\title{
Coherent-Pulse 2D Crystallography Using a Free-Electron Laser X-Ray Source
}

\author{
A. P. Mancuso, ${ }^{1}$ A. Schropp,,${ }^{1,}$ B. Reime, ${ }^{1}$ L.-M. Stadler, ${ }^{1}$ A. Singer, ${ }^{1}$ J. Gulden, ${ }^{1}$ S. Streit-Nierobisch, ${ }^{1}$ C. Gutt, ${ }^{1}$ \\ G. Grübel, ${ }^{1}$ J. Feldhaus, ${ }^{1}$ F. Staier, ${ }^{2}$ R. Barth, ${ }^{2}$ A. Rosenhahn, ${ }^{2}$ M. Grunze, ${ }^{2}$ T. Nisius, ${ }^{3}$ T. Wilhein, ${ }^{3}$ D. Stickler, ${ }^{4}$ \\ H. Stillrich, ${ }^{4}$ R. Frömter, ${ }^{4}$ H.-P. Oepen, ${ }^{4}$ M. Martins, ${ }^{5}$ B. Pfau, ${ }^{6}$ C. M. Günther, ${ }^{6}$ R. Könnecke, ${ }^{6}$ S. Eisebitt, ${ }^{6}$ B. Faatz, \\ N. Guerassimova, ${ }^{1}$ K. Honkavaara, ${ }^{1}$ V. Kocharyan, ${ }^{1}$ R. Treusch,,${ }^{1}$ E. Saldin, ${ }^{1}$ S. Schreiber, ${ }^{1}$ E. A. Schneidmiller,${ }^{1}$ \\ M. V. Yurkov, ${ }^{1}$ E. Weckert, ${ }^{1}$ and I. A. Vartanyants ${ }^{1, \dagger}$ \\ ${ }^{1}$ Deutsches Elektronen-Synchrotron DESY, Notkestraße 85, D-22607 Hamburg, Germany \\ ${ }^{2}$ Angewandte Physikalische Chemie, Universität Heidelberg, Im Neuenheimer Feld 253, D-69120 Heidelberg, Germany \\ ${ }^{3}$ Institute for X-ray-Optics, RheinAhr-Campus Remagen, FH Koblenz, Südallee 2, D-53424 Remagen, Germany \\ ${ }^{4}$ Institut für Angewandte Physik, Universität Hamburg, Jungiusstraße 11, D-20355 Hamburg, Germany \\ ${ }^{5}$ Institut für Experimentalphysik, Universität Hamburg, Luruper Chaussee 149, D-22761 Hamburg, Germany \\ ${ }^{6}$ Berliner Elektronenspeicherring-Gesellschaft für Synchrotronstrahlung m.b.H. (BESSY), \\ Albert-Einstein-Straße 15, D-12489 Berlin, Germany
}

(Received 24 September 2008; published 22 January 2009)

\begin{abstract}
Coherent diffractive imaging for the reconstruction of a two-dimensional (2D) finite crystal structure with a single pulse train of free-electron laser radiation at $7.97 \mathrm{~nm}$ wavelength is demonstrated. This measurement shows an advance on traditional coherent imaging techniques by applying it to a periodic structure. It is also significant that this approach paves the way for the imaging of the class of specimens which readily form $2 \mathrm{D}$, but not three-dimensional crystals. We show that the structure is reconstructed to the detected resolution, given an adequate signal-to-noise ratio.
\end{abstract}

DOI: 10.1103/PhysRevLett.102.035502

Revealing the structure of protein molecules is mandatory for understanding the structure of larger biological complexes. At the forefront of modern structural investigations is the knowledge of the functionality of biological systems on different length and time scales. The major progress in uncovering the structure of proteins in past decades was due to the development of phasing methods [1] allowing the determination of the structure of complex molecules that crystallize. In spite of considerable progress in macromolecular crystallography, crystallization and radiation damage is still a bottleneck in protein structure determination.

One new approach to overcome these difficulties is based on the use of ultrashort pulses of X-ray free-electron lasers (XFEL) that are presently under construction in the U.S., Japan, and Europe [2]. An elegant idea is based on measuring a sufficiently sampled diffraction pattern from a single molecule illuminated by an FEL pulse [3]. Femtosecond single pulse imaging was recently demonstrated [4-6] at the FLASH Facility in Hamburg [7]. However, in spite of the extreme intensity of the FEL pulses, a diffraction pattern from only one molecule will not be sufficient to obtain a high resolution diffraction pattern. Many reproducible copies will need to be measured to get a sufficient signal-to-noise ratio for each projection necessary for three-dimensional (3D) imaging at subnanometer spatial resolution. These noisy diffraction patterns will necessarily be of random orientation and the classification of these patterns is not straightforward $[8,9]$ and requires further development.
PACS numbers: 61.05.C-, 41.60.Cr, 42.30.Rx, 87.15.B-

Alternatively, we propose to use two-dimensional (2D) finite crystals to reveal the structure of single molecules. It is well known that a 2D infinite crystal cannot exist due to logarithmically growing fluctuations [10]; however, there are no limitations for the existence of $2 \mathrm{D}$ finite crystals. As a result, all molecules, or any other biological complexes arranged in a 2D crystal with identical unit cell structure, will give an enhanced signal in a single shot diffraction pattern from FEL sources. This can be especially important for the membrane proteins that in general do not form 3D crystals, but easily form 2D crystalline structures [11,12]. This approach is particularly attractive as the enhancement of the signal-to-noise ratio due to the periodicity improves as the square root of the number of repeated unit cells [13]. In addition, the unique opportunity to exploit an extension of this approach by collecting diffraction patterns from reproducible $2 \mathrm{D}$ crystals at different known orientations is possible. This enables complete 3D imaging of biological samples.

Coherent x-ray diffractive imaging (CXDI) has recently been developed [14-18]. The basic procedure involves a finite sized object being illuminated with coherent radiation, and its corresponding far-field diffraction pattern is measured, and importantly, sampled sufficiently. Given the knowledge of the object's finite extent, known as its finite support, the measured diffraction pattern can be inverted uniquely [19] using phase retrieval methods [20,21] to obtain an image of the object. The advantages here are the lack of optics required to make the measurement, and that the possible resolution is limited in principle only by 
diffraction, and in practice only by the signal-to-noise ratio in the diffraction measurement. This freedom from the resolution restrictions of conventional x-ray microscopy becomes increasingly important as the length scale of features in the samples studied decreases. CXDI with application to $2 \mathrm{D}$ crystallography was earlier tested with a computer generated 2D crystal structure [22] and a finite periodic array of nanoislands [23]. Here we report on a proof-of-principle CXDI experiment on a 2D finite crystal structure.

Free-electron lasers are especially well suited for such coherent 2D crystallography. They provide femtosecond coherent pulses with extremely high power. Only the combination of all of these unique properties will allow the realization of $2 \mathrm{D}$ crystallographic $\mathrm{x}$-ray imaging on biological systems. Brilliant, ultrashort pulses could overcome the radiation damage problem [3] which is a severe limitation of conventional crystallography at third-generation synchrotron sources [24]. Higher luminosity and hence improved statistics for such experiments can be obtained by the use of pulse trains that can be provided by FLASH and the European XFEL [2,7].

In our experiment FLASH [7] was operated in a regime producing 21 bunches of electrons per pulse train, with a pulse train repetition rate of $5 \mathrm{~Hz}$. The bunches within each pulse train were spaced at $1 \mathrm{MHz}$. The average pulse energy was $15 \mu \mathrm{J}$ which is equivalent to $6 \times 10^{11}$ photons per pulse or $1.3 \times 10^{13}$ photons per train at the source.

We demonstrate finite crystallography by using a crystal array that was prepared on a $100 \mathrm{~nm}$ thick $\mathrm{Si}_{3} \mathrm{~N}_{4}$ membrane coated with $600 \mathrm{~nm}$ of $\mathrm{Au}$, and $200 \mathrm{~nm}$ of Pd. The finite crystal sample was manufactured by milling holes in the film in a regular array pattern using a Focused Ion Beam (FIB). The "unit cell" of our crystal consists of a large hole of $500 \mathrm{~nm}$ diameter (representing a "heavy atom" in conventional crystallography) and a smaller hole of $200 \mathrm{~nm}$ diameter (representing a "light atom"). The cen- ters of these holes were separated by $495 \mathrm{~nm}$. The whole structure is composed of five unit cells in each direction, making the total structure size about $10 \mu \mathrm{m} \times 10 \mu \mathrm{m}$.

The diffraction data were measured at FLASH on the PG2 monochromator beam line [25] with a fundamental wavelength of $7.97 \mathrm{~nm}$. The monochromator was used in specular reflection (zeroth order) mode. The last mirror of the beam line is a focusing mirror with long focal length $(\geq 2 \mathrm{~m})$ which provides an image of the source [25]. We estimate a beam size in the focal plane of the order of $150 \mu \mathrm{m}$ FWHM in the horizontal and vertical directions with a flux of $4.5 \times 10^{10} \mathrm{ph} /$ pulse determined by ray tracing.

The experiment was conducted in a dedicated CXDI vacuum chamber [26] which was connected to the PG2 beam line. The chamber consists of an upstream shutter, a sample stage, and a flight tube to the charged-coupled device (CCD). The FEL beam was incident on the sample at a distance of $71.5 \mathrm{~m}$ from the source. The diffracted radiation then propagated $535 \mathrm{~mm}$ to the detector position (see Fig. 1). The detector used was an in-vacuum CCD (PI-MTE 2048B) with $2048 \times 2048$ pixels, each $13.5 \mu \mathrm{m}$ square with 16-bit digitization. For a $10 \mu \mathrm{m}$ sample size with our experimental conditions we found that far-field conditions are well satisfied and the diffraction pattern is adequately sampled with a sampling rate of 30 in each dimension. We used a $0.2 \mathrm{~s}$ exposure time to collect a series of single pulse train data from our sample with a coherent flux on the sample area of $1.5 \times 10^{10}$ photons per pulse train. This is an order of magnitude higher than the expected coherent flux of about $3 \times 10^{9}$ photons on the same sample area for the same exposure time at a thirdgeneration synchrotron source [27]. A beamstop was positioned in front of the CCD to protect the camera from the direct beam. A typical data set is shown in Fig. 2(a). It demonstrates an excellent visibility for our 2D finite crystal structure of $10 \mu \mathrm{m}$ size. By comparing the visibility

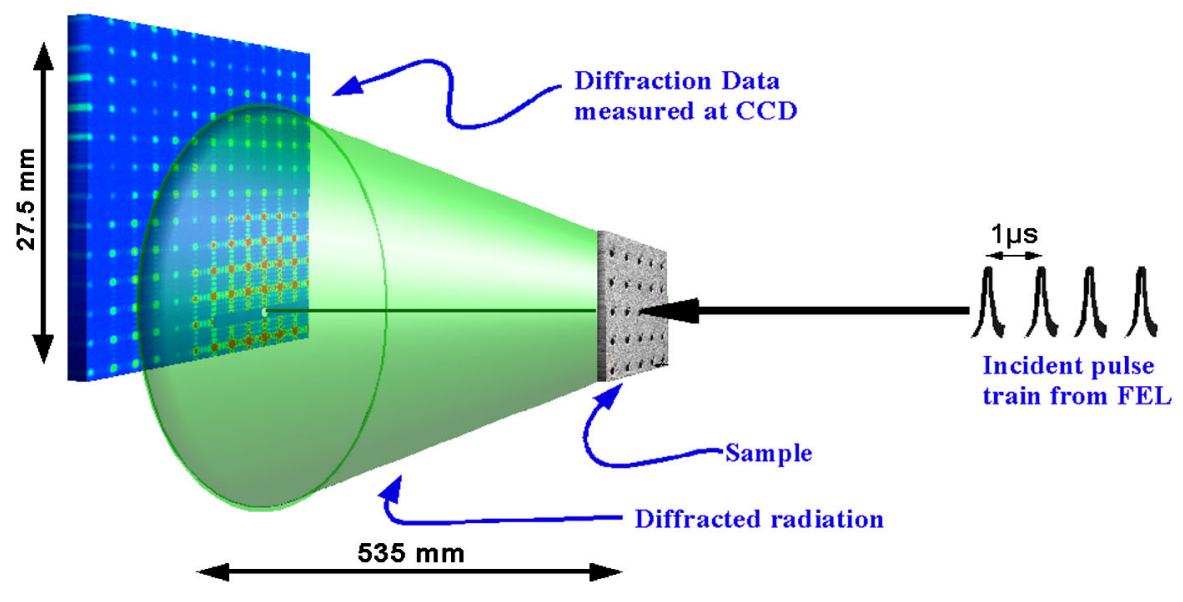

FIG. 1 (color online). A sketch of the experiment. The beam from the beam line first interacts with the sample, and then the diffracted radiation propagates to a CCD detector. To enhance the measured resolution of the data the direct beam was incident near the corner of the detector. 

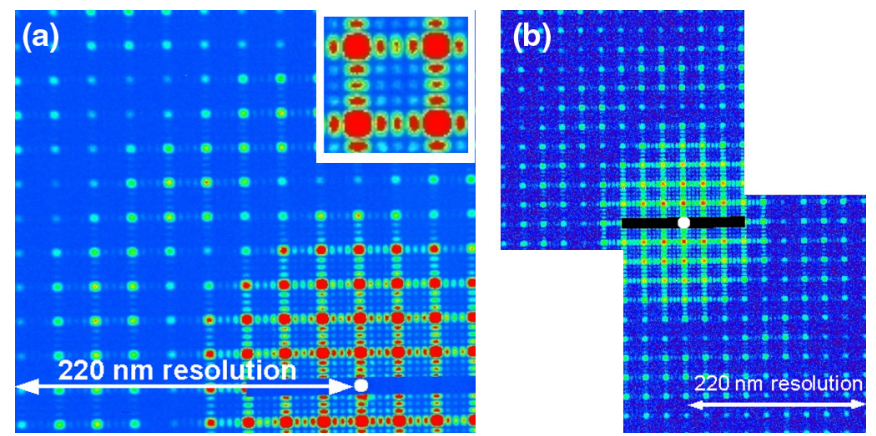

FIG. 2 (color online). (a) Far-field diffraction data measured from a single train of 21 femtosecond pulses from the FEL. (Inset) Enlarged region of diffraction pattern. (b) A symmetrized version of (a) used for reconstruction. The region in black corresponds to missing data covered by the beamstop. The white dot (in both panels) corresponds to the center of the incoming beam.

(98\%) of the measured data with a simulated diffraction pattern we conclude that the incident beam is fully coherent across the spatial extent of our sample.

The diffraction pattern in Fig. 2(a) contains signal up to the edge of the detector, which corresponds to a minimum feature size of $220 \mathrm{~nm}$. All expected features of a finite, crystalline structure are observed. The Bragg peaks due to the regular array are clearly seen, as are the oscillations between the Bragg peaks that are the result of the finite extent and coherent illumination of our sample. Also seen is the form factor from the individual elements- the large holes - that can be observed as a radial intensity modulation across the pattern.

To perform a reconstruction from the measured diffraction pattern shown in Fig. 2(a) several steps were implemented. The raw data were first corrected for background effects by subtracting dark field images. The data were then symmetrized about the center, and the pattern was binned $(3 \times 3)$ which also improves the signal-to-noise ratio of the measured data [Fig. 2(b)]. Symmetrizing the data is justified by the assumption that the sample is an amplitude object (as the incident radiation is totally blocked except at the holes), and consequently the measured diffraction pattern must be centrosymmetric [28]. After symmetrizing and binning, the data were $967 \times 967$ pixels in size. We embedded this array in a larger $2048 \times 2048$ pixel array to avoid artifacts in the reconstruction due to aliasing effects. The effective pixel size of this new array was $51.5 \mathrm{~nm}$ (which is clearly not the achieved resolution value as the data was measured to a resolution of $220 \mathrm{~nm}$ ). The regions without measured data (the beamstop, and the region outside the original $967 \times 967$ pixel area) were allowed to freely evolve during the reconstruction without additional constraint.

This preprocessed data set [Fig. 2(b)] was used for reconstruction by applying the hybrid input-output (HIO) iterative phase retrieval algorithm [20]. A scanning ion micrograph (SIM) image of our sample is shown in Fig. 3(a). The initial square support used for reconstruction is indicated by a dashed line in Fig. 3(b). For the reconstructed image shown in Fig. 3(c) we have used 1000 iterations of the HIO algorithm with the square support and the positivity constraint. It is clearly seen that the periodic array of large holes are successfully reconstructed; however, the small holes of $200 \mathrm{~nm}$ diameter are missing. Note also the diffuse, elongated background present in the reconstruction.

We have performed noise-free simulations of the reconstruction with the same region of missing diffraction data as in the experiment and with the same reconstruction parameters. These simulations clearly show the smallest $200 \mathrm{~nm}$ features that were missing in our initial reconstructions from the experimental data; however, they still exhibit the diffuse background seen in Fig. 3(c) which we attribute to the effect of the rather large beamstop on the reconstruction process.

Further simulations were performed with increasing levels of noise present in the simulated diffraction data. These data sets were created by adding a normally distributed random variable to our noise-free simulation. The standard deviation $\sigma$ of this distribution was proportional to the data value $I_{i}$ at each pixel $i: \sigma=\beta I_{i}$, where $\beta$ is the constant of proportionality, and $1 / \beta$ is the signal-to-noise
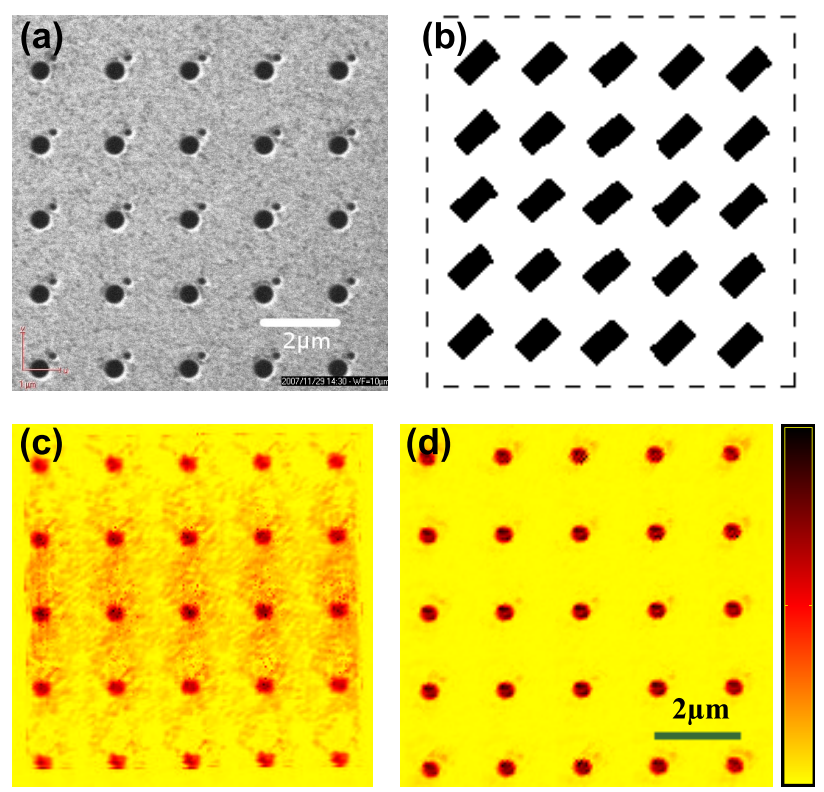

FIG. 3 (color online). (a) SIM image of the finite, periodic structure. (b) Support region used for the reconstruction of the diffraction pattern. The large rectangular support (dashed line) was used in the reconstruction shown in (c). The support in the form of 25 rectangular boxes was used in the reconstruction shown in (d). (c) The initial reconstructed image with the original data binned $3 \times 3$. (d) The final reconstructed image using the original data binned $5 \times 5$. The scale bar is $2 \mu \mathrm{m}$ in length. 
ratio. We performed reconstructions from these noisy diffraction patterns. Our simulations have shown that the data set with $\beta=5 \%$ reproduces the smallest features in the reconstruction. However, when the level of noise reaches $\beta=10 \%$ the dots of $200 \mathrm{~nm}$ size are no longer distinguished.

The noise levels of the experimental data were related to the simulated noise by fitting the intensity at a given Bragg peak by a function that corresponds to the diffraction pattern of a finite $2 \mathrm{D}$ crystal with $5 \times 5$ unit cells [29]. Our analysis has shown that the level of noise for the $3 \times 3$ binned data was about $\beta=7 \%$. Having achieved visibility in simulation of the smallest features only at $\beta=5 \%$, we obviously do not reconstruct them with this data set. One important question that we addressed as well in this simulation was the expected noise level for the same experimental conditions but just for only one unit cell as the sample. Our analysis has shown that for single unit cell parameter $\beta=14 \%$, which will be too high to obtain an ultimate resolution in reconstruction.

One possible explanation of this sensitivity to noise is that the measured diffraction pattern has two equivalent, symmetric solutions. One is with the small dots appearing to the top right of the larger dots, and the other is with them appearing to the bottom left. Because of this symmetry the reconstruction algorithm does not fully converge, but rather stagnates with two equivalent solutions superimposed with each other (compare with the same result obtained in [23]). As the measured data are highly oversampled we can bin the data further and in this way improve the signal-to-noise ratio. We have binned the data $5 \times 5$ (yielding a sampling rate of 6 in each dimension), and have obtained a characteristic noise level of about $\beta=2 \%$, that is, well below the critical value of $\beta=$ $5 \%$ [30]. In addition, instead of using a large square support we use a more constrained support of 25 rectangular boxes (similar to that used in [23]) each centered on the positions of the unit cell [see Fig. 3(b)] obtained in the previous reconstruction. The individual support boxes are of a significantly larger size than the reconstructed structures. By improving the noise statistics and reducing the symmetry in real space we were able to improve the reconstruction to the level that we resolved the smallest features present in our sample [Fig. 3(d)]. The reconstruction was performed by applying 2000 iterations of HIO with this fixed support.

Figure 3(d) shows that the 2D finite crystal structure is readily reconstructed from a single pulse train of the FEL beam. From line scans taken at the edge of a large hole in Fig. 3(d) we find that the variation in intensity from $10 \%$ to $90 \%$ of its maximum value occurs over less than four real space pixels (59.6 nm size) which yields a resolution of better than $238 \mathrm{~nm}$. This compares favorably with our measured maximum momentum transfer that corresponds to $220 \mathrm{~nm}$ resolution (see Fig. 2) where we still observe clearly defined features at the edge of the measured diffraction data.

In summary we have demonstrated single pulse train coherent diffractive imaging for a finite crystalline sample with the reconstructed image exhibiting resolution commensurate with the maximum momentum transfer measured in the diffraction data. Furthermore, we demonstrate that the traditionally noncrystalline framework of CXDI is applicable to two-dimensional finite crystals. In this experiment the $2 \mathrm{D}$ crystalline structure has been essential in providing the necessary signal to determine the structure of the unit cells. If only a single unit cell were used simulations suggest that a successful reconstruction to the resolution shown here would be impossible. These findings improve the scope of the fast growing CXDI technique by including finite $2 \mathrm{D}$ crystalline specimens in the sphere of applicability of CXDI, and by utilizing these crystals to improve signal to noise, and hence resolution of reconstructed images. We conclude that this alternative approach to single molecule imaging is a significant step towards revealing the structure of proteins with subnanometer resolution at the newly built XFEL sources.

*Present address: Technische Universität Dresden, Institut für Strukturphysik, Zellescher Weg 16, D-01062 Dresden, Germany.

Corresponding author. ivan.vartaniants@desy.de

[1] M. Woolfson and F. Hai-fu, Physical and Non-Physical Methods of Solving Crystal Structures (Cambridge University Press, Cambridge, 1995).

[2] J. Arthur et al., http://www.slac.stanford.edu/LCLS/CDR; T. Tanaka and T. Shintake, http://www-xfel.spring8.or.jp/ SCSSCDR.pdf; M. Altarelli et al., http://xfel.desy.de/tdr/ index_eng.html.

[3] R. Neutze et al., Nature (London) 406, 752 (2000).

[4] H. N. Chapman et al., Nature Phys. 2, 839 (2006).

[5] H. N. Chapman et al., Nature (London) 448, 676 (2007).

[6] A. Barty et al., Nat. Photon. 2, 512 (2008).

[7] W. Ackermann et al., Nat. Photon. 1, 336 (2007).

[8] G. Huldt et al., J. Struct. Biol. 144, 219 (2003).

[9] G. Bortel and G. Faigel, J. Struct. Biol. 158, 10 (2007).

[10] L. D. Landau and E. M. Lifshits, Statistical Physics (Reed Educational and Professional Publishing Ltd., Oxford, 2000), 3rd ed., Pt. 1.

[11] E. E. Uzgiris and R. D. Kornberg, Nature (London) 301, 125 (1983).

[12] Y. Kimura et al., Nature (London) 389, 206 (1997).

[13] The enhancement of the diffracted signal is proportional to the number of unit cells $N$. The noise in each pixel due to its statistical nature is proportional to the square root of the intensity. We see that the improvement in the signal-tonoise ratio increases with the square root of $N$.

[14] J. Miao et al., Nature (London) 400, 342 (1999).

[15] M. Pfeifer et al., Nature (London) 442, 63 (2006).

[16] H. N. Chapman et al., J. Opt. Soc. Am. A 23, 1179 (2006). 
[17] G. J. Williams et al., Phys. Rev. Lett. 97, 025506 (2006).

[18] D. Shapiro et al., Proc. Natl. Acad. Sci. U.S.A. 102, 15343 (2005).

[19] R. H. T. Bates, Optik 61, 247 (1982).

[20] J. R. Fienup, Appl. Opt. 21, 2758 (1982).

[21] V. Elser, J. Opt. Soc. Am. A 20, 40 (2003).

[22] J. Miao and D. Sayre, Acta Crystallogr. Sect. A 56, 596 (2000).

[23] I. A. Vartanyants and I. K. Robinson, J. Synchrotron Radiat. 10, 409 (2003).

[24] C. Nave and E. F. Garman, J. Synchrotron Radiat. 12, 257 (2005).

[25] M. Wellhoefer et al., J. Opt. A Pure Appl. Opt. 9, 749 (2007).

[26] B. Reime et al., in HASYLAB Annual Report 2006, edited by W. Caliebe, W. Drube, and J. R. Schneider (HASYLAB at DESY, Hamburg, 2006) Pt. 1, p. 1403.

[27] This estimate is based on a coherent flux of $10^{12}$ photons per second with the same incident photon energy and beam size as in our experiment at a third-generation synchrotron. Our estimate implies that measurements using FELs can be performed in less than one tenth of the collection time required by a synchrotron and orders of magnitude less in exposure time due to the femtosecond FEL pulses. This is especially important in the case of radiation sensitive biological materials.

[28] The biological systems to be studied with pulsed FEL beams in the future will be phase rather than amplitude objects. With the exception of symmetrizing the data set the results obtained in our present research can be extended to phase objects.

[29] J. Als-Nielsen and D. McMorrow, Elements of Modern $X$-Ray Optics (John Wiley \& Sons, New York, 2001).

[30] Improving the signal-to-noise level of a measured diffraction pattern with binning is a general procedure that can be used in the analysis of discretely sampled data. Clearly, the final diffraction pattern must be sampled finer than the Nyquist frequency [19]. 